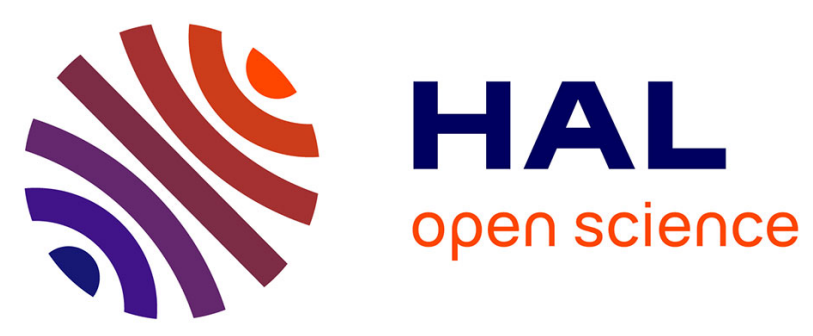

\title{
Phenotyping Thermal Responses of Yeasts and Yeast-like Microorganisms at the Individual and Population Levels: Proof-of-Concept, Development and Application of an Experimental Framework to a Plant Pathogen
}

Anne-Lise Boixel, Ghislain Delestre, Jean Legeay, Michaël Chelle, Frederic Suffert

\section{- To cite this version:}

Anne-Lise Boixel, Ghislain Delestre, Jean Legeay, Michaël Chelle, Frederic Suffert. Phenotyping Thermal Responses of Yeasts and Yeast-like Microorganisms at the Individual and Population Levels: Proof-of-Concept, Development and Application of an Experimental Framework to a Plant Pathogen. Microbial ecology, 2019, 78 (1), pp.42-56. 10.1007/s00248-018-1253-6 . hal-02159596

\author{
HAL Id: hal-02159596 \\ https://hal.science/hal-02159596
}

Submitted on 12 Aug 2020

HAL is a multi-disciplinary open access archive for the deposit and dissemination of scientific research documents, whether they are published or not. The documents may come from teaching and research institutions in France or abroad, or from public or private research centers.
L'archive ouverte pluridisciplinaire HAL, est destinée au dépôt et à la diffusion de documents scientifiques de niveau recherche, publiés ou non, émanant des établissements d'enseignement et de recherche français ou étrangers, des laboratoires publics ou privés. 
Author's Post-print: final draft post-refereeing

Publisher's Version: Microbial ecology, 78(1), 42-56

DOI: https://doi.org/10.1007/s00248-018-1253-6

Phenotyping thermal responses of yeasts and yeast-like microorganisms at the individual and population levels: proof-of-concept, development and application of an experimental framework to a plant pathogen

Anne-Lise BOIXEL ${ }^{1}$, Ghislain DELESTRE ${ }^{1}$, Jean LEGEAY ${ }^{1}$, Michaël CHELLE ${ }^{2}$, Frédéric SUFFERT ${ }^{1}$

${ }^{1}$ UMR BIOGER, INRA, AgroParisTech, Université Paris-Saclay, 78850, Thiverval-Grignon, France

${ }^{2}$ UMR ECOSYS, INRA, AgroParisTech, Université Paris-Saclay, 78850, Thiverval-Grignon, France

\section{Abstract}

Deciphering the responses of microbial populations to spatiotemporal changes in their thermal environment is instrumental in improving our understanding of their eco-evolutionary dynamics. Recent studies have shown that current phenotyping protocols do not adequately address all dimensions of phenotype expression. Therefore, these methods can give biased assessments of sensitivity to temperature, leading to misunderstandings concerning the ecological processes underlying thermal plasticity. We describe here a new robust and versatile experimental framework for the accurate investigation of thermal performance and phenotypic diversity in yeasts and yeast-like microorganisms, at the individual and population levels. In addition to proof-of-concept, the application of this framework to the fungal wheat pathogen Zymoseptoria tritici resulted in detailed characterisations for this yeast-like microorganism of: (i) the patterns of temperature-dependent changes in performance for four fitness traits; (ii) the consistency in thermal sensitivity rankings of strains between in planta and in vitro growth assessments; (iii) significant interindividual variation in thermal responses, with four principal thermotypes detected in a sample of 66 strains; (iv) the ecological consequences of this diversity for populationlevel processes through pairwise competition experiments highlighting temperature-dependent outcomes. These findings extend our knowledge and ability to quantify and categorise the phenotypic heterogeneity of thermal responses. As such, they lay the foundations for further studies elucidating local adaptation patterns and the effects of temperature variations on ecoevolutionary and epidemiological processes.

Keywords: phenotyping, responses to temperature, thermal performance curve, diversity metrics, yeast-like microorganisms, Zymoseptoria tritici 


\section{I - INTRODUCTION}

Microorganisms inhabit diverse environments differing considerably in terms of their temperature conditions (mean, range, variance, dynamics). For example, foliar pathogens live in patchy environments subject to thermal variations, the magnitude and rate of which vary over space (heterogeneity) and time (dynamics) [1,2]. Temperature has a large effect on the growth of microorganisms, and is thus a crucial factor that must be taken into account when deciphering the ecological and epidemiological processes in which these microorganisms are involved [3], e.g. disease development for foliar pathogens of plants [4]. The response to thermal conditions differs between individuals [3]; therefore, it is vital to characterise the diversity of these responses in natural populations to predict changes, such as epidemic outbreaks, particularly in a context of climate change [5]. To that end, a set of efficient, reproducible and standardised thermal phenotyping protocols is necessary to quantify these responses accurately and to ensure that they can be compared between individuals, populations and experiments.

In addition to the fact that protocols meeting these requirements are still lacking to date [6], the phenotyping of thermal responses is currently subject to six types of limitation. These limitations may be inherent to the experimental design (i-ii), or may arise in either the measurement (iii-v) or data analysis (vi) phases namely:

- (i) suboptimal choice of the proxy for fitness when investigating phenotypic heterogeneity between individuals in the absence of prior multiple trait-based approaches. Indeed, the fitness traits under study will not necessarily have a similar effect on the components of performance (growth, reproduction and survival) or be affected similarly by temperature [7, 8];

- (ii) fragmented range and disparate resolution of the thermal conditions studied, leading to errors in the prediction of key thermal parameters [9];

- (iii) potential biases in the measurement of performance likely to magnify or counteract differences between replicates and/or individuals. This can be seen in the case of fungi, for which conventional protocols rely on radial growth rate measurements on solidified agar surfaces in Petri dishes. Indeed, colony diameter and area do not capture growth in three dimensions and are affected by both the physical heterogeneity of the moisture content of the solid medium and the differences in surface tension between plates [10];

- (iv) suboptimal consideration and monitoring of time-dependent effects inherent to labile fitness traits [11], with measurements often conducted at a single time point;

- (v) use of an incorrect indicator of the temperature actually perceived by the microorganism during the experiment (e.g. micro- vs. phyllo-climate [2]). For example, air temperatures are widely used in studies carried out under controlled (incubator or phytotron units), semicontrolled (greenhouse) or natural conditions (field) but do not correspond to the temperatures of the immediate environment of microorganisms i.e. culture medium (in vitro assays) or leaf (in planta assays) temperatures [4];

- (vi) incorrect description of the experimental data when establishing TPCs [12] (thermal performance curves, which depict the variation of performance over a range of temperatures [7]) due to the inappropriate selection of candidate models (e.g. a linear model with only two temperatures) and/or an underappreciation of the constraints of the model on the confidence estimates of key thermal parameters [13].

All these factors result in a partial view of the relationship between trait, fitness and temperature, by hindering the plotting of accurate and robust TPCs. 
Based upon these limitations, the design of phenotyping protocols is a potential stumbling block in the development of future high-throughput phenotyping assays assessing the phenotypic heterogeneity of thermal responses. This is particularly evident in the case of Zymoseptoria tritici (formerly Mycosphaerella graminicola), the causal agent of Septoria tritici blotch (STB), one of the most important foliar diseases of wheat worldwide [14]. For this biological model, research on thermal adaptation has been restricted to in vitro comparisons of two temperatures, with growth measured on solidified agar, at one to three time points $[10,15,16]$, resulting in the inherent biases mentioned above. A phenotyping protocol has been proposed in planta for the latent period [4], defined as the time interval between infection and sporulation, but it is suitable only for small numbers of samples (time-consuming and intensive procedures for disease assessment over a wide range of temperatures). Furthermore, in the case of in planta assessments, differential responses of performance (the expression of aggressiveness in this instance) between strains can take the form of a cross effect between host resistance and temperature adaptation $[17,18]$.

This study aimed to bridge these gaps by providing a generic experimental framework for phenotyping the responses of a microorganism to its temperature at the individual (responses of quantitative traits to temperature) and population (interindividual variability for a given trait) levels. This framework was designed to be suitable for use with microorganisms whose growth predominantly results from budding or fission (e.g. yeasts and yeast-like microorganisms). It was implemented here for $Z$. tritici, to provide both proof-of-concept and new research findings concerning the thermal responses of this pathogen.

We present in this study: (i) a laboratory-standardised and miniaturised method for accurately characterising the full functional thermal responses of $Z$. tritici over a wide range of temperatures, with practical recommendations for its use for yeasts and other yeast-like microorganisms; (ii) an investigation of the interdependence of $Z$. tritici phenotypic traits regarding individual thermal sensitivity (phenotypic integration), to assess the biological relevance and quality of the information acquired in this framework; (iii) a characterisation of the diversity of thermal responses in $Z$. tritici between individuals and within and between populations; (iv) an assessment of the consequences of this diversity for population-level processes.

\section{II - METHODS}

The responses of $Z$. tritici to its thermal environment were analysed by establishing TPCs for sampled strains under laboratory-standardised and miniaturised conditions. This method involves measuring differences in growth kinetics (i.e. the increase in the number of yeast-like spores over time) within aliquots of different spore suspensions exposed to 12 temperatures (II.1). We assessed its veracity (II.2) and biological relevance (II.3), and then used it to investigate the diversity of thermal phenotypes in $Z$. tritici and its consequences for population-level processes (II.4).

\section{A STEP-BY-STEP GUIDE TO THE THERMAL PHENOTYPING FRAMEWORK}

An in vitro approach (to eliminate the interaction between $Z$. tritici and wheat plants) was developed to screen a large number of strains, using growth kinetics as a proxy for fitness. The steps in the thermal phenotyping process are summarised in Fig. 1. 
Preparation of fungal cultures (Fig. 1.1) - Inoculum was prepared from stock tubes of $Z$. tritici spore suspensions stored at $-80^{\circ} \mathrm{C}$ in a $1: 1$ glycerol-water mixture. Subcultures were grown on PDA (potato dextrose agar, 39 g. $\mathrm{L}^{-1}$ ) in a Petri dish and kept at $18{ }^{\circ} \mathrm{C}$ in the dark for six days. Spores were scraped from the agar surface and resuspended in a glucose peptone liquid medium (14.3 g.L $\mathrm{L}^{-1}$ dextrose, 7.1 g.L $\mathrm{L}^{-1}$ bactopeptone and 1.4 g.L $\mathrm{L}^{-1}$ yeast extract). Concentrations of the resulting suspensions were adjusted to $2.5 \times 10^{5}$ spores. $\mathrm{mL}^{-1}$ in a Malassez counting chamber. For each spore suspension, eight culture aliquots were dispensed in the eight wells of a column of a 96-well microtiter plate (Microtest Plate, Sarstedt), with an eight-channel pipette $(150 \mathrm{\mu L}$ per well). The first column was reserved for the blank (eight wells containing noninoculated liquid medium), to take into account the contribution of the medium to optical density. With our experimental design, it was possible to study 11 strains simultaneously on each microtiter plate of a given experimental series. Microtiter plates were prepared in 12 replicates, each of which was assigned to one of the 12 temperatures studied. Plates were sealed with a sterile gas-permeable membrane (Breathe-Easy, Diversified Biotech) to prevent contamination and liquid medium evaporation. Preliminary tests were conducted to select the optimal culture conditions for studying $Z$. tritici growth at various temperatures (see ESM 1a).

Initiation of the growth experiment (Fig. 1.2) - Each plate was statically incubated in the dark for four days at one of the 12 study temperatures from 6.5 to $33.5{ }^{\circ} \mathrm{C}(6.5,9.5,11.5,14.5$, $17.5,20.0,22.5,24.5,26.5,28.5,30.5$ and $33.5^{\circ} \mathrm{C}$ ). The number, resolution and range of these temperatures were chosen so as to ensure the accurate capture of responses to temperature [9]. We chose to stop monitoring growth at four days for two reasons: to capture the growth kinetics as precisely as possible until saturation (stationary phase) whilst avoiding the phase during which experimental artefacts would be likely to occur. A biofilm-like structure [19] formed at the surface of the liquid medium after five days, and this structure altered spore number estimates in the microplate wells (see ESM 1b). The 12 constant thermal environments were obtained by placing the plates in plastic boxes in an incubator (Heratherm Incubator IMC 18, Thermo Fisher Scientific) or in a water bath under thermostatic control (Polystat 24 Immersion Circulator, Thermo Fisher Scientific).

Kinetic monitoring of fungal growth (Fig. 1.3) - Plates were removed from their thermal environment to measure the optical density of each well at $405 \mathrm{~nm}\left(\mathrm{OD}_{405}\right)$ three times per day, at five-hour intervals, with a microplate spectrophotometer (Multiskan FC and dedicated SkanIt Software, Thermo Fisher Scientific). Plates were shaken before each reading (horizontal shaking for 5 seconds at $5 \mathrm{~Hz}$, with an amplitude of $15 \mathrm{~mm}$ ) to aerate the medium and ensure a uniform distribution of spores within the suspension.

Establishment of TPCs - Optical density $\left(\mathrm{OD}_{405}\right)$ measurements were converted into spore numbers for each suspension, with a calibration curve (see Eq. 1). This calibration curve, which relates $\mathrm{OD}_{405}$ values to the actual spore concentration of 30 dilutions (1.25x dilution series, as recommended in [20]), was established for four reference strains and was shown to be suitable for broad-spectrum application when assessed with a set of 68 strains (no significant difference in curve coefficients).

$$
\boldsymbol{O D}=4.2 \times 10^{-7} \boldsymbol{S}+0.06(E q .1)
$$

where $\boldsymbol{O D}$ is the $\mathrm{OD}_{405}$ value subtracted by the intrinsic absorbance of the medium (blank value) and $\boldsymbol{S}$ is the number of spores per millilitre estimated with an automated particle counter (FlowCell FC200S+, Occhio). 
The TPC for a given strain was obtained by: (i) fitting logistic curves to its growth kinetics curve at each of the 12 temperatures studied (Fig. 1.4); (ii) extracting the mean maximum growth rate for each fitting (Fig. 1.5); (iii) fitting a non-linear model to biological growth rate data (Fig. 1.6). Since the goodness-of-fit of models to data varies with temperature (i.e. on the $\mathrm{x}$ axis of TPCs), the systematic errors in predictions of performance may differ between models at a given temperature. Thus, as population-based studies are more focused on deciphering interindividual variations in TPCs than in obtaining absolute performance values for each strain, we decided to use a single model to describe TPCs for a given trait. After initial fitting tests on the experimental data which was obtained for Z. tritici, 15 mathematical models were chosen from a set of 50 models published in previous studies as descriptors of trait responses to temperature (see ESM 2). These 15 models were classified according to their goodness and statistical relevance of fit to experimental data. The criteria for discriminating between models were the residual sum of squares, and the Akaike and Schwarz weights [21]. We then selected the best of the top-ranking candidate models by focusing successively on: (i) the distribution of residuals across the temperature range, with greater weight given to the estimation accuracy of mid-range rather than extreme temperatures; (ii) model constraints, including in particular dependence of parameters [22] and symmetry [12]; (iii) practicality, i.e. whether the model has biologically meaningful parameters and is frequently used to describe TPCs in the literature (see ESM 2).

Capturing key thermal parameters from the TPC (Fig. 1.7) - The most appropriate model was adjusted separately for each strain studied. The characteristics of each TPC were captured with four key thermal parameters: maximum performance $\left(\mathrm{P}_{\max }\right)$, thermal optimum $\left(\mathrm{T}_{\text {opt }}\right)$, maximum temperature $\left(\mathrm{T}_{\max }\right)$ and thermal performance breadth $\left(\mathrm{TPB}_{80}\right.$ : temperature range over which performance exceeded $80 \%$ of $\left.\mathrm{P}_{\max }\right)[3,7]$.

\section{METHOD VALIDATION}

The method was validated by assessing the accuracy, repeatability, reproducibility and suitability of the thermal phenotyping framework as defined by ISO international standards [23, 24]. The framework phenotyping steps (see Fig. 1) were applied to two Z. tritici reference strains (CBSKNAW-IPO323 and INRA-FS0932) in an experiment that was repeated three times consecutively (repetitions of experiment). For both strains, spore concentrations were measured for eight aliquots (technical replicates) at each of the 13 time steps for the analysis of growth kinetics with two methods: turbidity (OD) and automated (Flow-Cell FC200S+ particle counter and dedicated CALLISTO software, Occhio) measurements. The veracity of growth estimates from $\mathrm{OD}_{405}$ measurements was evaluated by assessing agreement and precision between optical density (OD method) and particle counter (PC method) measurements. As extreme temperatures can modify spore melanisation $[16,25]$ and as $Z$. tritici displays dimorphism (potential transition from yeastlike to hyphal growth forms, depending on growth conditions [26]), we also checked that changes in OD were not due to shifts in the optical properties of spores during growth monitoring. Spore size (length and width), shape (straightness), melanisation (greyscale level) and growth form (visual discrimination procedure) were analysed from morphometric data and images of all spores passing through the particle counter (accuracy assessment; see ESM 3a). Differences in spore concentrations were investigated between the technical replicates for each set of growth conditions (strain $\mathrm{x}$ time $\mathrm{x}$ temperature) and between the three independent and consecutive 
repetitions of the experiment (repeatability and reproducibility assessments, respectively). We ensured that thermal growth conditions were homogeneous in the wells of each microtiter plate, by carrying out a specific experiment at $11.5,18.0$ and $24.5{ }^{\circ} \mathrm{C}$, respectively: 20 T-type thermocouples (copper-constantan; diameter $0.2 \mathrm{~mm}$ ) were immersed in the culture medium of 20 wells and connected to a datalogger (Campbell Scientific), which recorded temperature every 10 minutes (suitability assessment; see ESM 4).

\section{CONSISTENCY IN THERMAL SENSITIVITY FOR ASSESSMENTS BASED ON FITNESS TRAITS}

Is the thermal sensitivity inferred from in vitro growth rate representative of in planta traitbased thermal responses? 'Phenotypic integration' [27] of thermal responses was studied for a set of 18 Z. tritici strains in liquid medium (in vitro) and on wheat plants (in planta). In vitro assessment involved monitoring the growth kinetics of each strain at 12 temperatures (see Fig. 1), whereas in planta assessment was based on three conventional aggressiveness traits (AUDPC, latent period and sporulation capacity; see Table 1). Each strain was inoculated by applying a spore suspension (calibrated at $2 \times 10^{5}$ spores. $\mathrm{mL}^{-1}$ ) to the median part of the flag leaf (adaxial face) of six adult wheat plants ( $c v$. Apache, head fully emerged stage) [28]. This experiment was conducted simultaneously under five thermal regimes at mean temperatures of $12.4( \pm 0.4), 14.3$ $( \pm 0.5), 17.1( \pm 0.4), 18.9( \pm 0.4)$ and $21.5( \pm 0.4){ }^{\circ} \mathrm{C}$. Leaf temperature was recorded throughout the experiment with T-type thermocouples positioned under the leaf in contact with the inoculated area [4]. Disease symptoms were quantified twice weekly until total leaf senescence, through visual assessments of the sporulating area and the quantification of spore production [28], to provide information about in planta traits (see Table 1). For each trait, TPCs were established with the best mathematical model from 15 candidates (see ESM 2). Based on these TPCs, we conducted (i) a multi-trait analysis to assess matches in thermal response patterns between the four traits; (ii) a consistency assessment in which we ranked strains based on the position of TPCs on the temperature axis for growth rate (in vitro) and AUDPC (in planta).

\section{PRACTICAL APPLICATIONS}

\section{Application 1: Capturing thermal phenotypic diversity between individuals and within and between populations}

How much do thermal responses vary between individuals and populations of $Z$. tritici? Six $Z$. tritici populations of 11 strains each, collected on wheat $c v$. Apache in fields located along two transects in France (a north-south increasing gradient of mean annual temperature and a westeast increasing gradient of annual temperature range; see ESM 5a), were phenotyped. The TPCs of all strains were established with a modified version of the thermal phenotyping framework (growth measured $72 \mathrm{~h}$ after deposition i.e. shortly after reaching the inflection point in the growth kinetics) and were summarised by the calculation of $\mathrm{P}_{\max }, \mathrm{T}_{\mathrm{opt}}, \mathrm{T}_{\max }$ and $\mathrm{TPB}_{80}$. Overall thermal phenotypic diversity was assessed by establishing an average TPC template and determining the extent to which it varied between strains. We then conducted a hierarchical clustering on principal components (HCPC) analysis on $\mathrm{P}_{\max }, \mathrm{T}_{\text {opt }}, \mathrm{T}_{\max }$ and $\mathrm{TPB}_{80}$, to group together thermal responses with similar characteristics (entities hereafter referred to as 'thermotypes'). We then summarised this diversity, using the corresponding clusters silhouette plot [29] to visualise the number, proportion and features (compactness, separation, connectivity) 
of each thermotype. We assessed the variation of TPCs within and between populations, by analysing both divergence in the means and variances of thermal parameters and the composition patterns of thermotypes.

Application 2: Inferring relative fitness at the individual (performance) and population (competitive advantage) levels - TPCs show how temperature affects a given component of fitness (applied here to growth rate) at the individual level. Can we upscale this knowledge to group or population levels using additive rules? We conducted a pairwise competition experiment to determine whether the actual relative fitness of two strains grown together at a given temperature can be inferred from their respective individual TPCs. Five $Z$. tritici strain pairs, selected on the basis of their (dis)similar TPCs, were cocultured in $150 \mathrm{\mu L}$ of GPL, with an initial total spore concentration of $2.5 \times 10^{5}$ spores. $\mathrm{mL}^{-1}$, at eight temperatures $\left(7.0,11.5,15.0,18.5,21.0,25.0,28.5\right.$ and $\left.30.5{ }^{\circ} \mathrm{C}\right)$, in five replicates. This proof-of-concept experiment involved the monitoring of changes in strain frequencies $72 \mathrm{~h}$ after deposition, by measuring the final proportions of each strain by fluorescence microscopy and image analyses [30] (see Fig. 2). Strain pairs were constituted by coculturing a GFP-expressing IPO323 transformant (IPO323* strain, $\mathrm{T}_{\text {opt }}=25.8{ }^{\circ} \mathrm{C}$ ) with one of the following non-GFP-expressing strains: (i) a strain with a similar TPC and genetic background (WT-IPO323 corresponding to IPO323* wild-type, $\mathrm{T}_{\text {opt }}=25.8^{\circ} \mathrm{C}$ ) at initial proportions of 25,50 and $75 \%$ in the coculture; (ii) a strain with a similar TPC but a contrasting genetic background (FS0917, $\mathrm{T}_{\text {opt }}=25.7^{\circ} \mathrm{C}$ ) at an initial proportion of 50\%; (iii) three strains (analyses conducted separately) with similar TPCs but differing from IPO323* (FS0678, FS0922, FS1014; $\mathrm{T}_{\text {opt }}=19.1,19.2,19.3{ }^{\circ} \mathrm{C}$, respectively), at an initial proportion of $50 \%$ (see Fig. 7a).

\section{STATISTICAL ANALYSES}

Four types of statistical analyses were conducted with $\mathrm{R}$ software [31]: (i) comparisons of means between groups based on analysis of variance $(A N O V A)$ or its non-parametric alternative (Kruskal-Wallis test); followed, when necessary, by appropriate post hoc tests ( $P$-value threshold: 0.05); (ii) fitting and analysis of a generalised linear model quantifying the extent to which OD values may be affected by experimental variables and their interactions with time and temperature (II.2); (iii) nonlinear fitting and selection of mathematical models to establish TPCs [32]; (iv) exploratory multivariate analyses (PCA and HCPC) [33] to define thermotypes, the characteristics of which were explored further with clustering validation indices [34] (II.4.1).

III - RESULTS

\section{METHOD VALIDATION}

\section{Veracity and precision of spore concentration measurements}

The results obtained with the OD and PC methods displayed a high degree of agreement, as shown by the strong correlation between paired measurements (Pearson's correlation coefficient: 0.99; $P<0.01$ ), although the OD method tended to give slightly higher estimates (by 0 to $7 \%$ of the total number of spores in the sample). The OD method displayed less variation in spore number estimates between successive replicate measurements (Student's $t$-test; $P<0.01$ ) and, as such, appeared to be more reliable for the detection of small but significant differences between strains (see ESM 6). 
The OD method is repeatable but requires normalisation in sequential test series An ANCOVA was performed on the log-transformed growth kinetics of IPO323 and FS0932 (see Fig. 3). Spore concentration values were expressed as the combined effect of strain $(P<0.01)$, OD reading time $(P<0.01)$, technical replicates $(P=0.99)$, well position (core or edge wells; $P$ $=0.95)$ and repetitions of experiment $(P<0.01)$. In other words, the OD method was highly repeatable, with no significant variation in spore concentration between aliquots or between positions on the microtiter plate. However, consecutive repetitions of the experiment were not strictly equivalent. Thus, when the set of strains cannot be phenotyped in a single batch, a control (reference strain) should be grown in each experimental run for subsequent normalisation.

\section{The OD method is suitable for the thermal phenotyping of Z. tritici}

We assessed the homogeneity of the system in terms of temperature and potential biological artefacts, to check that the phenotyping results obtained with the OD method were truly representative. We first checked the thermal homogeneity of in vitro growth conditions between wells of the same microtiter plate at three temperatures (deviation of the temperature of individual wells from the overall mean temperature of the microtiter plate $<$ maximum residual error of measurements; see ESM 4b). Regarding spore optical properties during growth monitoring, spore length, width and shape had no significant effect on the change in OD values. Suspensions exposed to extreme temperatures (below $11.5^{\circ} \mathrm{C}$ and above $30.5{ }^{\circ} \mathrm{C}$ ) contained a slightly higher proportion of hyphal forms (not statistically significant). This change in the proportion of hyphal forms, which remained below $1 \%$ in all experimental conditions (see ESM $3 b)$, had no effect on OD at any temperature except at $9.5^{\circ} \mathrm{C}$ (interaction Hyphae:Temperature; $P=0.02$ ), at which the hyphal forms presented more signs of branching filamentous structures. Melanisation affected OD, with a gradual increase in melanin levels detected after day 2 of the experiment (see ESM 3c), but this effect was not dependent on the temperature of the liquid medium (see Table 2 and ESM 3d).

\section{CONSISTENCY IN THERMAL SENSITIVITY FOR ASSESSMENTS BASED ON DIFFERENT FITNESS TRAITS}

\section{Difference in TPCs between the four fitness traits}

The mean TPCs for in vitro growth rate, AUDPC, LAT and nbSPO were described by different non-linear functions and key thermal parameter values (see Fig. 4). These differences reflect variations of the shape and position of performance distribution along the temperature axis (e.g. $\mathrm{T}_{\text {opt }}$ was $4.3{ }^{\circ} \mathrm{C}$ higher for in vitro growth rate than for AUDPC; 20.3 vs. $16.0{ }^{\circ} \mathrm{C}$ ), with large differences in the rate and magnitude of changes in performance per degree change in temperature over the range. The overall correlation between in vitro growth rate and latent period or sporulation capacity was weak, but the one between in vitro growth rate and AUDPC was strong, for $\mathrm{T}_{\mathrm{opt}}$ values (see Fig. 5b) and for full responses (Pearson's correlation coefficient: global $\left.0.76, \mathrm{~T}_{\text {opt }} 0.92 ; P<0.05\right)$.

\section{Consistency of the thermal sensitivity rankings of strains for in vitro growth rate and AUDPC}

In vitro growth rate was found to be a reliable proxy for the assessment of thermal sensitivity, with the results obtained highly consistent with aggressiveness in planta. As TPC shape did not differ significantly between the 18 strains (Kruskal-Wallis, $P<0.05$ ), $\mathrm{T}_{\text {opt }}$ can be considered to reflect the position of the TPC along the temperature axis. This parameter was used to rank 
strains for both in vitro growth rate and AUDPC (see Fig. 5a). In total, 11 of 18 strains were assigned to the same thermal sensitivity rank, and the ranking errors between the two assessments ranged from -2 to +2 . Consequently, strain categorisation (e.g. 'cold-adapted' vs. 'warm-adapted' strains) was conserved whether estimated in vitro or in planta. A clear benefit of the in vitro method is the magnification of the $\mathrm{T}_{\mathrm{opt}}$ distribution relative to in planta disease assessment (4.8 vs. $2.2{ }^{\circ} \mathrm{C}$; see Fig. 5c). This difference was particularly pronounced for coldadapted strains, as there was a slight imbalance (distribution tail of the kernel density plot) in $\mathrm{T}_{\text {opt }}$ distribution for lower values.

\section{APPLICATION 1: CAPTURING THERMAL PHENOTYPIC DIVERSITY BETWEEN INDIVIDUALS AND WITHIN AND BETWEEN POPULATIONS}

\section{Classification and description of thermal responses: modes of variation and thermotypes}

The beta distribution was the preferred candidate model for describing TPCs for in vitro growth rate as it provided the most accurate and efficient description of the thermal response of this trait (see ESM 2). Therefore, this model was used to establish the mean TPC template and determine the degree to which it varied between strains (see Fig. 6a). Interindividual differences in TPCs were significant across all strains for the parameters $\mathrm{P}_{\max }, \mathrm{T}_{\text {opt }}$, and TPB TANOVA, $P$ $<0.05)$. $\mathrm{T}_{\max }$ was largely conserved across $Z$. tritici strains, with a confined value centered around $33.5 \pm 0.7^{\circ} \mathrm{C}$. The minimum temperature values $\left(\mathrm{T}_{\min }\right)$ given by the equation were not considered as they fell outside the range of experimental values $\left(6.5-33.5^{\circ} \mathrm{C}\right)$. Four thermotypes were identified across populations: Th1 ('adapted to warmer conditions'), Th2 ('specialist'), Th3 ('high performer') and Th4 ('generalist') (see Fig. 6b and Table 3).

\section{Compactness, separation and connectivity of thermotypes}

All TPCs discriminated well between the four thermotypes (classification conducted without assumptions and with all retained PCA dimensions) except for two strains with a negative or almost null silhouette width coefficient. Thermotypes were well separated (distance between cluster centers and between strains in different clusters), with Th3 and Th4 less differentiated from each other. All thermotypes displayed substantial within-cluster variations, which were larger for Th1 and Th2 (higher within-cluster distance metrics) and particularly well structured for Th3 (bimodal distribution of silhouette coefficients: two distinct groups at about 0.55 and 0.25 , respectively) (see Fig. 6c).

\section{Within and between population variation of TPCs}

A similar diversity of thermal responses (similar means and variances) was observed for $T_{\text {opt }}, T_{\max }$ and $\mathrm{TPB}_{80}$ between the six populations (ANOVA and Bartlett's test: $P>0.05$ ). The four thermotypes were represented in all populations except for Fp5, which was composed of Th1 and Th2 only. With a higher proportion of Th1 (poor-performance strains) and no Th3 (highly performant strains) in its population, Fp5 had a lower $\mathrm{P}_{\max }$ (ANOVA and Tukey HSD, $P<0.01$ ) due to the difference in thermotype composition compared with the other populations (see ESM $5 b)$. 


\section{APPLICATION 2: INFERRING RELATIVE FITNESS AT THE INDIVIDUAL AND POPULATION LEVELS}

\section{No competitive advantage for strains with similar TPCs}

The final proportion of IPO323* spores was not affected by temperature in coculture with WTIPO323 (ANOVA, $P>0.05$ ), regardless of the initial ratio (see Fig. 7i). Furthermore, no significant difference was found between the initial and final proportions of each strain (ANOVA, $P>0.05)$. This suggests that neither strain had a competitive advantage over the other. Likewise, the final proportion of IPO323* spores did not vary significantly over the temperature range in coculture with FS0917 (ANOVA, $P>0.05$ ). However, FS0917 slightly outcompeted IPO323*, with final spore proportions of $57 \%$ and $43 \%$ (ANOVA, $P=0.02$ ), respectively (see Fig. 7ii).

\section{Expression of a competitive advantage between strains with different TPCs}

The final proportion of IPO323* spores increased significantly along the temperature axis (Kruskal-Wallis, $P<0.01$ ) in coculture with FS0678, FS0922 or FS1014, whose TPCs are characterised by a lower $\mathrm{T}_{\text {opt }}\left(19.2 \pm 0.1\right.$ vs. $25.8^{\circ} \mathrm{C}$; see Fig. 7aiii). The same trend (described by a second-order polynomial equation) was observed for the three strains, indicating that the TPC dissimilarity of each strain with the TPC of IPO323* was responsible for the outcome of competition (see Fig. 7 biii).

\section{IV- DISCUSSION}

\section{PROPERTIES, APPLICATIONS AND SCALING-UP OF THE THERMAL PHENOTYPING FRAMEWORK}

The thermal phenotyping framework developed here satisfactorily overcomes the six common limitations of such phenotyping methods (see introduction). It is miniaturised (microtiter plate method), standardised (balanced and homogeneous growth conditions), accurate (more reliable spore quantification, yielding a slight overestimate), robust (sound repeatability and potential of comparison, if a reference strain is included in the experimental design of sequential test series for subsequent normalisation) and suitable for thermal phenotyping (no skewed confounding effect related to shifts in the optical properties of spores and magnification of the $\mathrm{T}_{\mathrm{opt}}$ distribution compared with in planta disease assessment). On this last point, dimorphism and melanisation, both of which have been reported to be temperature-dependent [25, 35], notably in $Z$. tritici [16], could have affected OD measurements. Consequently, they were taken into account in the definition of the validity range of the method. Spore melanisation was not involved in any confounding effect or interaction with temperature on changes in OD. This seeming contradictory result (absence of interaction between melanisation and temperature) with those presented in [16] likely stems from the substantial differences in our methodological approaches since the use of liquid medium and the particle counter allowed us to measure melanisation directly at the spore level and not at the single-spore colony level (inherent to the use of solid medium, with possible differential effects of the air-colony-medium interface on melanisation). Regarding $Z$. tritici dimorphism, temperature did not affect the proportion of hyphal growth forms, which can be explained by the optimised culture conditions of our framework (in particular consideration of the characteristics of culture medium regarding nutrient deprivation rate and colony ageing; see ESM 1), but shifts from the yeast-like to the hyphal growth form might significantly affect $\mathrm{OD}$ at low temperatures (i.e. around $9.5^{\circ} \mathrm{C}$ - as was the case in our experimental conditions - 
and probably below). This all the more supports our decision not to include $\mathrm{T}_{\min }$ values in our analyses of thermal responses given their low confidence levels.

This framework is a significant improvement over those used in previous studies investigating thermal adaptation in $Z$. tritici $[10,15,16]$. The use of a liquid medium makes higher-throughput screening assays possible (further design miniaturisation) and avoids the problems associated with physical differences between plates (moisture content and surface tension of solid medium), which can affect colony deposition and distort growth measurements [10]. It also covers a wide range of temperatures, with a finer-grained resolution, providing a more complete picture of thermal responses, which can be efficiently captured only by TPCs. In the case of $Z$. tritici, the use of 12 temperatures rather than two may overcome the problems associated with simple tests of 'thermal sensitivity' based on two temperatures $[10,15,16]$, in which it can be questionable to draw firm conclusions given the non-linearity of thermal responses. Indeed, depending on the position relative to $T_{\text {opt }}$ of the two temperatures considered, slope comparisons could lead to a misinterpretation of the results. Finally, growth rate measurements were not segregated at one to three single time points, but measured over four days to take time-dependent effects into account when reporting maximum growth rates inferred over the kinetics.

This framework can be applied to the study of other microorganisms whose spores grow exclusively through cell division (e.g. yeasts and yeast-like microorganisms in general), by following the step-by-step directions presented here: tailoring of the framework to the target microorganism (preliminary growth monitoring experiments to define the optimal growth conditions, the experimental time frame and the range and resolution of thermal conditions; see ESM 1), method validation (verification of accuracy, repeatability, reproducibility and suitability; see II.2) and a thorough assessment of the information obtained (cross-comparisons between the thermal responses of other fitness traits studied in the natural environment of the microorganism; see II.3).

\section{CAPTURING PLASTICITY IN Z. TRITICI THERMAL RESPONSES IN INDIVIDUALS AND IN POPULATIONS}

\section{Phenotypic variation in thermal responses within and between French populations}

- In this study, we quantified the intraspecific phenotypic variability of thermal responses within populations of a fungal pathogen with a high level of genetic and phenotypic diversity [36, 37]. Significant variation in TPCs between individuals was reported across strains from all six populations sampled. TPCs were classified into four thermotypes, each of which distinguished a particular category of thermal responses with its own intrinsic features (e.g. generalist or specialist strains) and differentiation (e.g. compactness and separation from other thermotypes on the basis of cluster distance metrics). This categorisation is a promising way to summarise diversity at different levels (individual, population, species). It should be noted that this constitutes not an absolute but a relative classification varying with the populations which are sampled. Given the differences in interindividual variation within thermotypes (e.g. scattered distribution for Th2 vs. bimodal distribution for Th3), thermal response diversity should be considered not only between, but also within thermotypes. This is of particular importance as the phenotypic composition of groups can drive the adaptation of a population to its environment through eco-evolutionary processes [38]. 
Spatial structure of thermal responses in French populations - No spatial phenotypic structure of thermal responses was detected between the six French Z . tritici populations, although one population displayed a considerably lower level of thermal response diversity (only two of the four identified thermotypes present). These populations were not sampled over the same time period during the growing season (at the end of winter for Fp5 and Fp6 and at the end of spring for Fp1, Fp2, Fp3 and Fp4). Since we are analysing a potentially blurred picture with seasonal variations as a confounding factor, it is not possible to conclude that there is absolutely no pattern of thermal adaptation in France. Indeed, diversity in the thermal responses of $Z$. tritici at the population level may emerge not only at a worldwide scale (significant differences in sensitivity to temperature linked to genetic differentiation [10]), but also locally (selection pressure exerted by seasonal changes [15]). Consequently, future investigations of phenotypic diversity in $Z$. tritici populations and its potential structuration by thermal environments should involve careful consideration of the most appropriate sampling strategy [39] along spatial and temporal gradients because the traits studied here are labile. The populations sampled over a same period namely winter (extreme points of the west-east increasing gradient of annual temperature range) and spring (north-south increasing gradient of mean annual temperature) populations can be analysed separately. Interestingly, the Fp5 population, which displays the lowest richness level of diversity in thermal responses (only two thermotypes), was devoid of the generalist thermotype compared to the Fp6 population in which we detected both the specialist and the generalist thermotypes. Fp5 is characterised by a marked distinction in the annual range of its thermal environment as its range was the lowest among all sampling locations especially when compared with Fp6 (11.9 vs. $19.9^{\circ} \mathrm{C}$, respectively, whilst they share the same annual thermal mean). This finding is consistent with the assumption that more variable environments favour thermal generalists [3]. Despite the difference in mean annual temperature along the north-south gradient, the same level of thermal response diversity and, in particular, a similar proportion of 'warm-adapted' strains were found in Fp1, Fp2, Fp3 and Fp4. This deviates from the assumption that there is a shift in the optimal range of thermal responses in relation to the mean temperature encountered within an environment. This high local diversity but nongeographically based population structure along the north-south gradient may be explained by the high gene flow among $Z$. tritici populations at large spatial scales due to the long-distance wind-dispersal of ascospores [36].

Differential effects of leaf temperature on aggressiveness traits - Leaf temperature effects on three fitness traits expressed in planta were studied through the establishment of TPCs, as described in a previous study focusing on latent period [4]. The knowledge of the range and distribution of TPC parameters acquired here for $18 \mathrm{Z}$. tritici strains could be incorporated into epidemiological models to investigate how and to what extent pathogen development and epidemic dynamics are affected by variations of temperature (potentially opposite effects of temperature on different aggressiveness traits; see Fig. 4) and the diversity of responses between individuals within a population (not accounted for in current models, despite the demonstration of a significant impact in epidemic forecasting [40]). A comprehensive multi-trait approach is particularly important because: (i) selection pressure does not necessarily operate on the same traits over the course of an epidemic [15]; (ii) strains can be regarded as patchworks of traits [41] with different levels of phenotypic plasticity that can be reliably estimated from the differences in TPC shape. 
Ranking consistency of thermal responses between in vitro and in planta assessments - Interestingly, the thermal optima for growth rate (in vitro) and AUDPC (in planta) were found to be strongly related. In particular, thermal sensitivity rankings were highly consistent whether estimated in vitro or in planta, but with an important shift in the thermal optimum and its distribution breadth. It is likely that this observed shift $\left(4.3{ }^{\circ} \mathrm{C}\right.$ higher for the in vitro growth rate optimum) results from the difference in the nutritional environment and in $Z$. tritici growth forms between in vitro culture (predominantly yeast-like growth) and in planta leaf medium (hyphal growth, from epiphytic growth on the leaf surface to the intercellular growth within plant tissues [26]) and/or from an effect of temperature on the host-pathogen interaction. This ranking consistency between in vitro and in planta assessments is in line with previous findings $[42,43]$ and suggests some sort of interdependence between phenotypic traits in individual thermal responses, consistent with the concept of phenotypic integration. This also constitutes an ultimate step of methodological validation considerably expanding the scope of the experimental framework by confirming its biological relevance, even though the results are acquired in vitro. Additionally, this finding has important implications as it suggests that relative ranking of thermal responses between strains might be sufficient to draw conclusions on the thermal adaptation of groups and populations based on rank-based analyses of variance. However, it should be underlined that studies aiming to assess and to project performance in response to changes in temperature continue to require inference from the full TPC (position, curvature and skew of the curve along the temperature axis).

\section{INFERRING FROM TPCs THE RELATIVE PERFORMANCE OF STRAINS IN} A THERMAL ENVIRONMENT

How well can overall performance and competitive advantage be predicted from TPCs? An extension of the thermal phenotyping framework (see application 2 in II.4) makes it possible to explore this question by inferring the performance of a given $Z$. tritici strain within a group or a population in a given thermal environment. We showed that the significance of these findings and the confidence that can be placed in them depend strongly on the properties of the experimental methods used (accuracy, repeatability, reproducibility, suitability of phenotyping assessment) and the correct integration of thermal biology and microbial ecology concepts. In our experiments, the relative proportions of the strains in pairwise cocultures of strains with similar TPCs were not affected by temperature regardless of genetic background. By contrast, pairwise cocultures of strains with dissimilar TPCs resulted in temperature-dependent outcomes with an increase in the proportion of the warm-adapted strains along the temperature axis. An expansion of this in vitro experiment with additional competition conditions (e.g. starting from different initial conditions and/or with more thermotypes in competition) would improve our understanding of the response of microbial populations to natural thermal variations. By extending the conditions (in planta) and the epidemiological framework (e.g. polycyclic dynamics) of the experiment, we could also try to improve characterisation of the thermal signal filter (time step and rate of the foliar fungal pathogen response to the environment [8]) and the speed of natural selection in constant or fluctuating thermal environments [44].

\section{FRAMEWORK VERSATILITY: APPLICATIONS FOR FUTURE RESEARCH}

This integrated framework from experimental design to data interpretation allows detailed phenotyping of the thermal responses of microorganisms. Through the quantification of interindividual phenotypic variation, it opens up new possibilities for exploring not only the 
diversity of thermal responses and its structuration (e.g. by highlighting patterns of local adaptation in natural populations [45]) but also its causes and consequences.

Indeed, this framework could help to provide deeper insight into the genetic basis of adaptation to temperature, by moving from phenotype to genotype (quantitative trait loci mapping or genome-wide association study of contrasting phenotypic profiles e.g. thermotypes) to identify genes involved in thermal adaptation (association of SNPs with given performance traits) [46] or from genotype to phenotype (phenotyping of specific temperature-sensitive allelic variants or mutant lines) to facilitate the functional annotation of genomes [47].

This framework can also be used to explore the consequences of phenotypic variation between individuals, through the use of TPCs to infer individual performance in a given population and thermal environment (see application 2 in II.4) and to predict changes in population composition in response to thermal changes [48], drawing on mark-release-recapture [49], selection [50] or modelling [51] experiments.

\section{CONCLUDING REMARKS}

By directly addressing the technical and analytical challenges in quantifying and describing thermal responses, this study provides a new methodology for inferring and categorising such responses in the case of yeasts and yeast-like microorganisms. As such, the proposed framework can assist in shedding more light on the phenotypic heterogeneity of thermal responses between individuals, populations, species and communities of these microorganisms, but also on the ways in which they interact with their natural or engineered environment. Among other applications, it can be used extensively to understand, predict and control the design, optimisation and conduct of food (e.g. strain selection), biomedical (e.g. product stabilisation), industrial (e.g. anaerobic digestion process for the production of biomethane), epidemiological (e.g. pathogen growth and epidemic development) and ecological processes (e.g. population dynamics in response to thermal variations). 


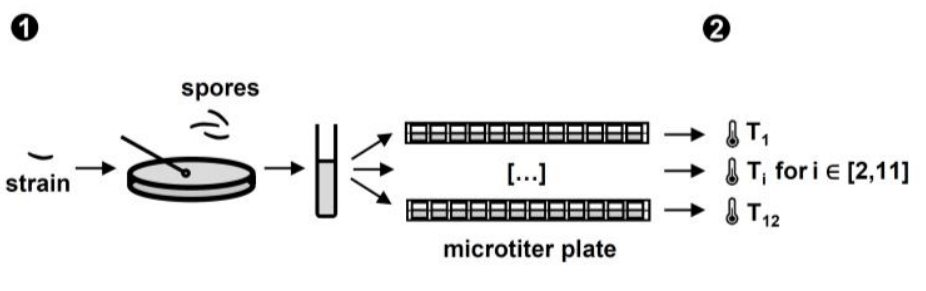

4

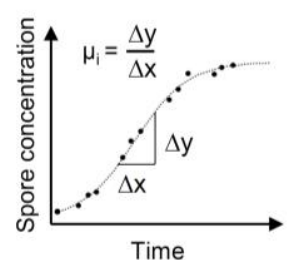

(5)

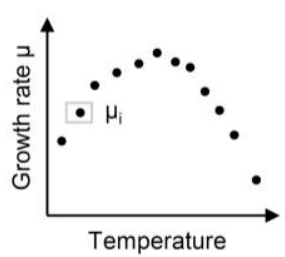

3

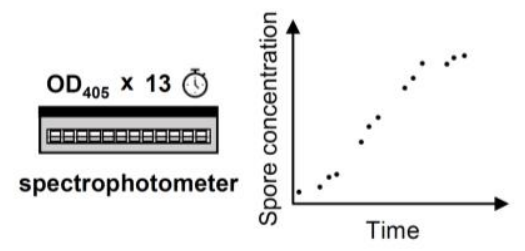

7

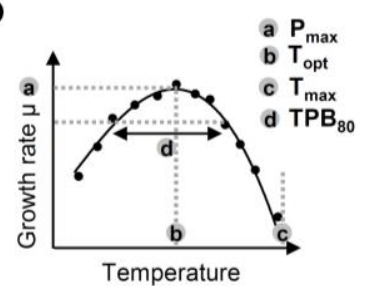

Fig. 1 Overview of the steps in the thermal phenotyping framework for a given strain. (1) Preparation of fungal cultures by adjusting spore suspensions and dispensing them in 12 microtiter plates; (2) Incubation of microtiter plates in the dark for four days at 12 temperatures; (3) Kinetic monitoring of fungal growth for each temperature through $\mathrm{OD}_{405}$ measurements with a spectrophotometer; (4) Calculation of the maximum growth rate $\mu$ (slope at the inflection point) for each temperature; (5) Expression of $\mu$ as a function of temperature; (6) Establishment of the strain's thermal performance curve (TPC) by the fitting of mathematical models to the growth rate data illustrated in step 5; (7) Capture of TPC features by retrieving key thermal parameters: maximum performance $\left(\mathrm{P}_{\max }\right)$, thermal optimum $\left(\mathrm{T}_{\text {opt }}\right)$, maximum temperature $\left(\mathrm{T}_{\max }\right)$ and $80 \%$ thermal performance breadth $\left(\mathrm{TPB}_{80}\right)$.

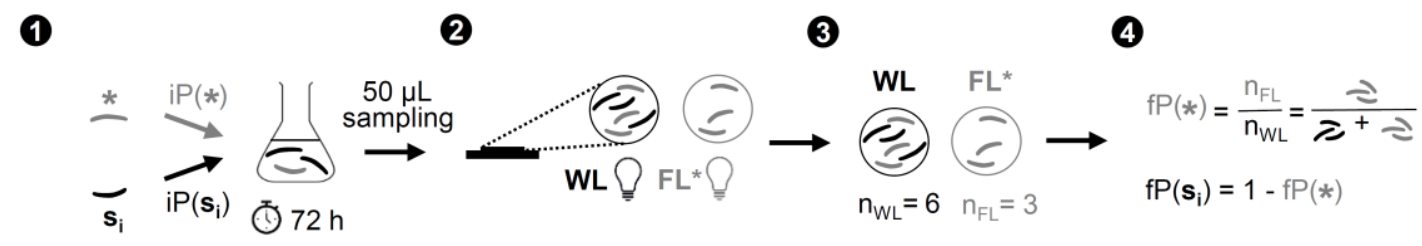

Fig. 2 Relative fitness measurements in pairwise competition experiments at eight constant temperatures. (1) We tested pairs of strains consisting of a GFP-expressing strain (IPO323* annotated as $*$ ), the spores of which were visible under both white (WL) and fluorescent $\left(\mathrm{FL}^{*}\right)$ light and a non-GFP-expressing strain $\left(\mathrm{s}_{\mathrm{i}}\right)$, the spores of which were visible only under WL. IPO323* spores were cocultured with $\mathrm{s}_{\mathrm{i}}$ spores at initial proportions $[\mathrm{iP}(*)]$ of 25, 50 and $75 \%$, in a 96-well microtiter plate (Microtest Plate, Sarstedt), with each well containing $150 \mu \mathrm{L}$ of inoculated GPL liquid medium. After 72 hours of growth, $50 \mu \mathrm{L}$ aliquots of each coculture replicate were sampled and deposited on a microscope slide; (2) Slides were photographed under both WL and $\mathrm{FL}^{*}$, at 10x magnification, with a fluorescence microscope equipped with a camera (Leica DM5500 B, Leica Microsystems) and LAS-AF software (Leica Microsystems). GFP fluorescence was captured with a filter, with excitation at 450-490 nm and emission in the 500-550 nm range; (3) All acquired images were analysed with Image $1.48 \mathrm{v}$ software. After conversion into black and white images, spores were counted on WL- $\left(\mathrm{n}_{\mathrm{WL}}\right)$ and FL*- $\left(\mathrm{n}_{\mathrm{FL}}\right)$ photographs with the built-in Subtract Background, Threshold and Analyze Particles functions; (4) Calculation of the final proportions (fP) of each strain in the coculture. 


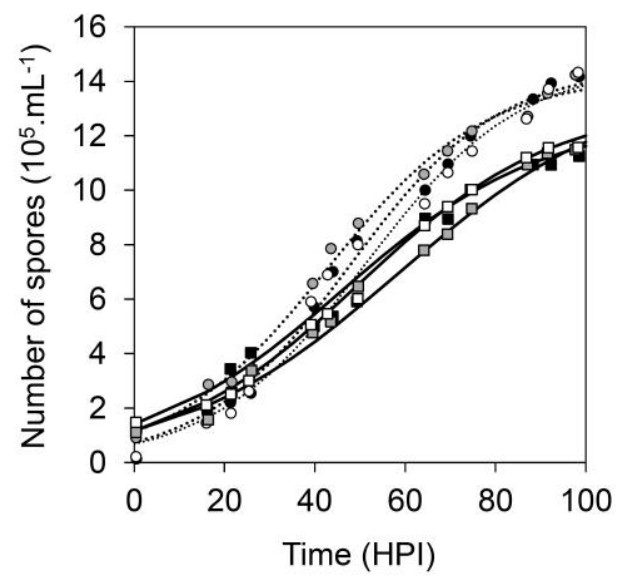

Fig. 3 Changes in spore concentration within the suspension over four days. Growth kinetics of two $Z$. tritici strains (here represented as a function of time in hours post-inoculation - HPI - and at their respective $\mathrm{T}_{\mathrm{opt}}$ ) were analysed by fitting a logistic regression model to the experimental data obtained for IPO323 (squares and solid lines) and FS0932 (circles and dotted lines). Each data point corresponds to the mean of eight technical replicates in an experiment repeated three times (each repetition being shown in a different colour).
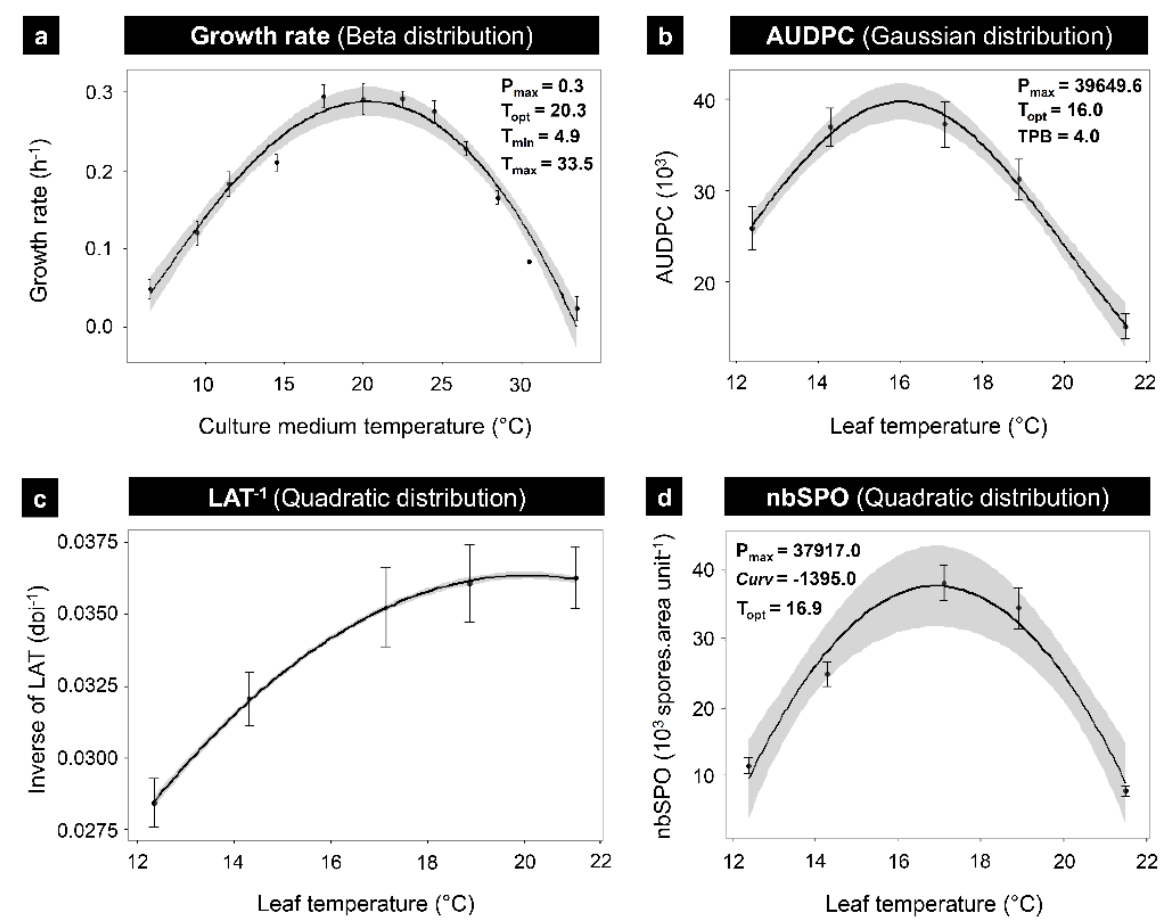

Fig. 4 TPCs of four $Z$. tritici phenotypic traits: (a) In vitro growth rate; (b) In planta AUDPC (area under the sporulating area progress curve); (c) Inverse of in planta latent period expressed in $\mathrm{dpi}^{-1}$ (number of days post-inoculation ${ }^{-1}$ required to reach $5 \%$ of the maximum sporulating area); (d) In planta sporulation capacity (final number of pycnidiospores produced per unit of maximum sporulating area). Data points correspond to the mean trait performance calculated for $18 \mathrm{Z}$. tritici isolates (mean $\pm \mathrm{SEM}$ ). TPCs were obtained by fitting the most appropriate mathematical model (solid line) to each data set and are displayed with their $95 \%$ confidence intervals (shaded area). 
a

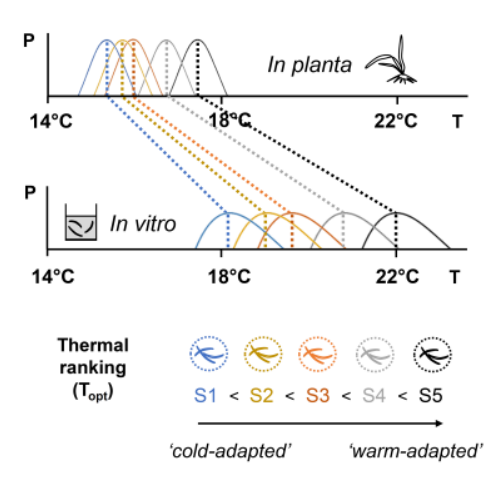

b

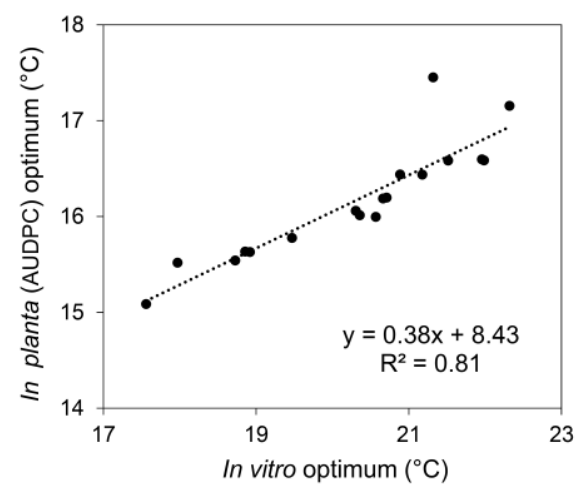

C

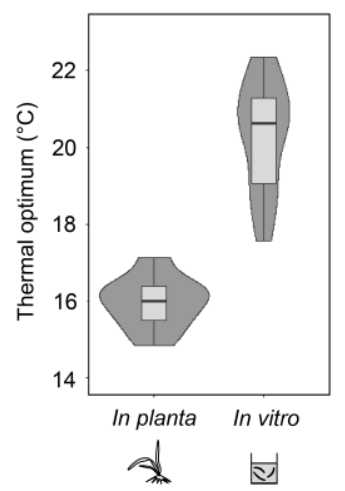

Fig. 5 Consistency in the ranking of thermal responses for 18 strains assessed on the basis of two phenotypic traits. (a) Theoretical representation of the information provided by the position of Topt (dotted lines) along the temperature axis (horizontal shift of TPCs showing performance $\mathrm{P}$ as a function of temperature $\mathrm{T}$ ) and consecutive thermal sensitivity rankings of five strains (S1-S5) for in vitro and in planta assessments; (b) Relationship between the in vitro growth rate Topt and the in planta AUDPC Topt of 18 Z. tritici strains illustrated by a linear model (dashed line) fitted to experimental data (points); (c) Full distribution (violin plot) of in planta AUDPC Topt (mean \pm SEM: $16.0 \pm 0.1{ }^{\circ} \mathrm{C}$ ) and in vitro growth rate Topt (mean \pm SEM: $20.3 \pm 0.3{ }^{\circ} \mathrm{C}$ ). 

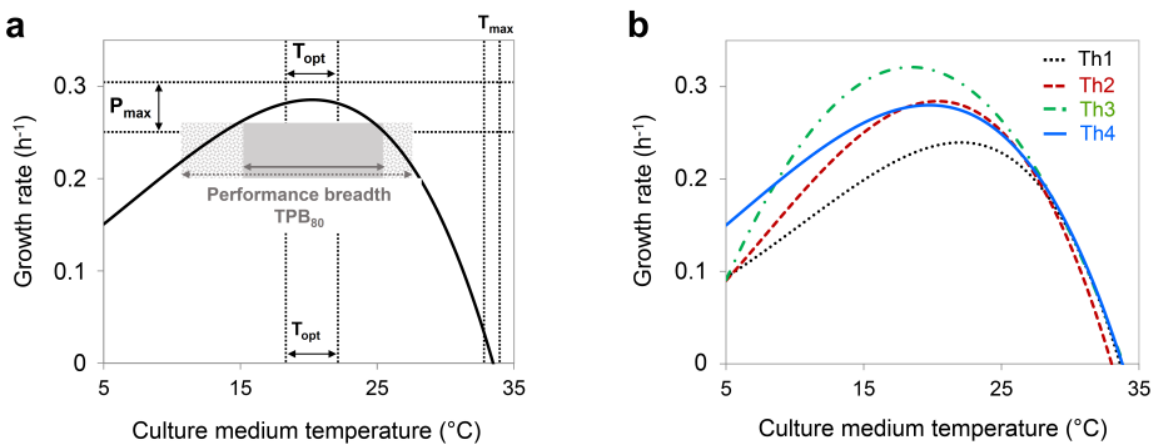

C
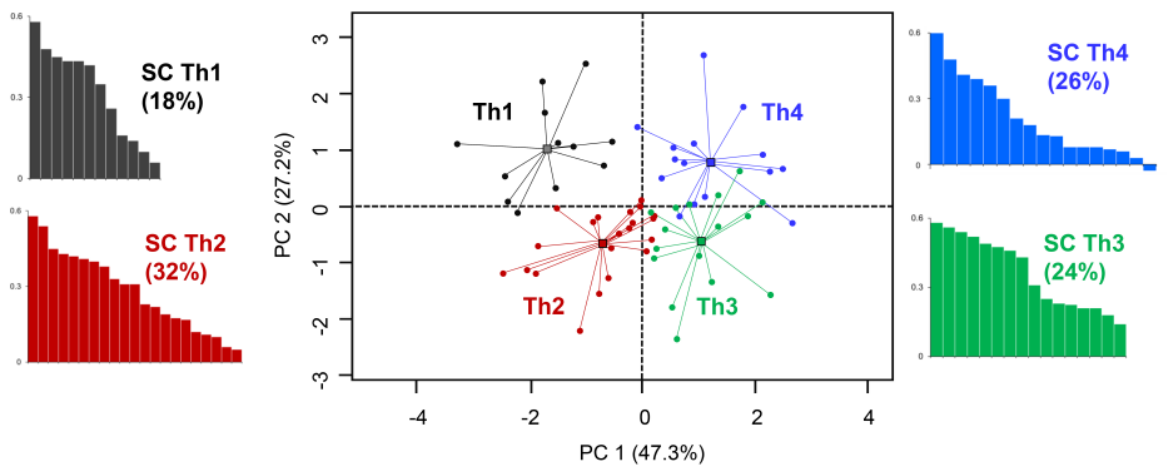

Fig. 6 Assessment of the diversity of $Z$. tritici thermal responses encountered within a set of six French populations ( 6 x 11 strains) collected along north-south and westeast transects. (a) Representative mean TPC obtained for in vitro growth rate and variation of its characteristics across strains (movement and shift in TPC position along the $x$ - and $y$-axes). The curve was obtained by fitting a beta distribution to the whole data set ( $n=66$ strains). Interindividual variation around the average function is displayed for four thermal parameters capturing TPC features: maximum performance $\left(\mathrm{P}_{\max }\right)$, thermal optimum $\left(\mathrm{T}_{\mathrm{opt}}\right)$, maximum temperature $\left(\mathrm{T}_{\max }\right)$ and $80 \%$ thermal performance breadth $\left(\mathrm{TPB}_{80}\right)$. Dotted lines and rectangles represent the lower and upper bounds of the standard deviation for each parameter. (b) Representative TPCs (paragons for each cluster) for each Z tritici thermotype (Th1: 'adapted to warmer conditions', Th2: 'specialist', Th3 'high performer' and Th4 'generalist') obtained by hierarchical clustering on principal components (HCPC) analysis for the four thermal parameters indicated above (see Table 3). (c) Clustering of thermal responses based on $\mathrm{P}_{\max }, \mathrm{T}_{\text {opt }}, \mathrm{T}_{\max }$ and $\mathrm{TPB}_{80}$. Factorial plan of the principal component analysis (PCA), with dots representing strains and coloured according to thermotype (HCPC cluster). The distance between each individual and the centre of the corresponding cluster is indicated by a line. Cluster silhouette plots (distribution of silhouette coefficient - SC - quantitative measurements of how well each strain lies within its cluster - for each individual of a given cluster) are also provided to illustrate and quantify disparities within (compactness) and between (connectivity) thermotypes. On these plots, the numbers in brackets refer to the proportion of the set of 66 strains corresponding to the thermotype concerned. 
i IPO323* vs WT-IPO323 $^{*}$
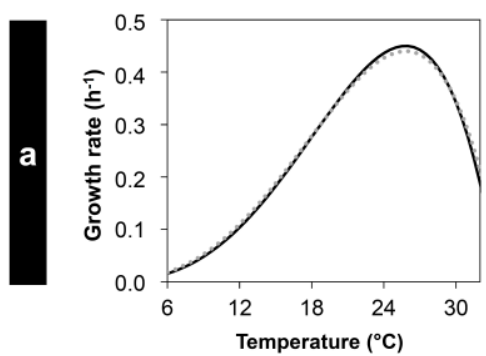

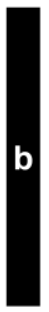

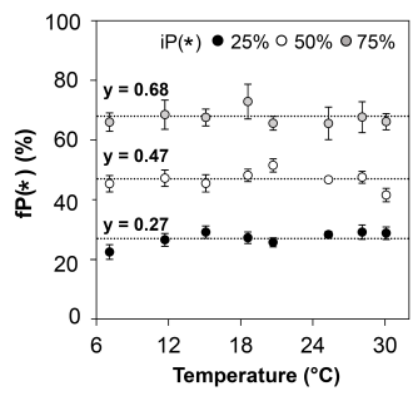

ii IPO323* vs FS0917
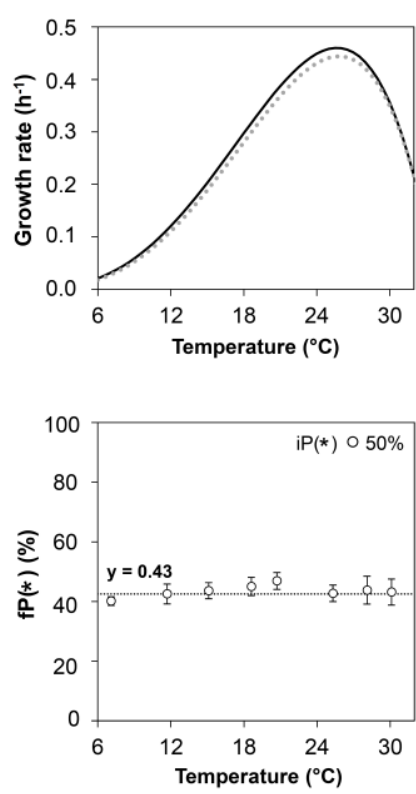

IP0323* vs FS1/FS2/FS3
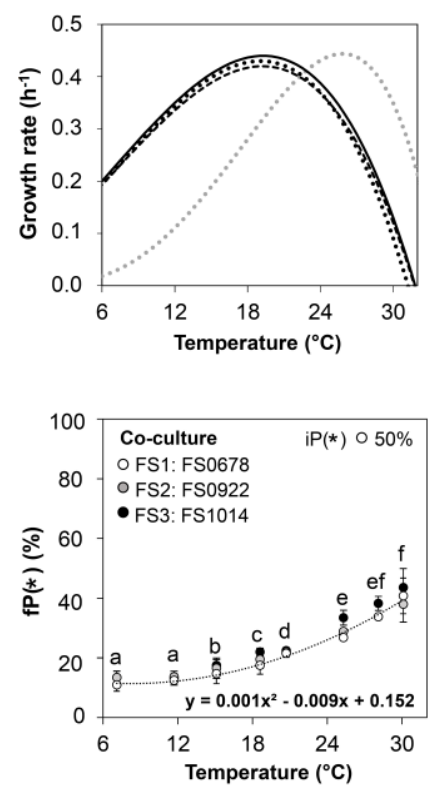

Fig. 7 Impact of individual TPCs on the outcome of pairwise competition between $Z$. tritici strains. (a) TPCs for in vitro growth rate for the five strains used in pairwise cocultures with IPO323* (GFP-expressing IPO323 transformant; $\mathrm{T}_{\text {opt }}=25.8{ }^{\circ} \mathrm{C}$; grey dotted curve). The various strains (black curves) paired with IPO323* have either (i) a matching TPC and identical genetic background (wild-type WT-IPO323; $\mathrm{T}_{\text {opt }}=25.8^{\circ} \mathrm{C}$ ) or (ii) a matching TPC but different genetic background $\left(\mathrm{FS} 0917 ; \mathrm{T}_{\mathrm{opt}}=25.7^{\circ} \mathrm{C}\right)$ or $(\mathbf{i i i})$ both a contrasting TPC and a different genetic background (FS1: FS0678, FS2: FS0922, FS3: FS1014; Topt $_{1}=19.1,19.2,19.3$ ${ }^{\circ} \mathrm{C}$, respectively). (b) Competition outcomes expressed as the final proportion of fluorescent spores (IPO323*), $\mathrm{fP}(*)$, obtained after the separate exposure of each coculture to eight liquid culture medium temperatures. Experimental data are presented as means (data points) \pm SEM (error bars). Dotted lines correspond to the trend line for change along the temperature axis for each initial proportion of IPO323*, iP $(*)$, i.e. 25, 50, 75\%, when applicable. Different letters above data points in Fig. 7biii indicate significant differences in final spore proportions as a function of temperature (Kruskal-Wallis test followed by post hoc pairwise comparisons, $P<$ $0.05)$. 


\section{TABLES}

Table 1 Definition, measurement and calculation of the three aggressiveness traits for which response to temperature was studied in the case of $18 \mathrm{Z}$. tritici strains

\begin{tabular}{|c|c|c|c|}
\hline Trait & AUDPC & \multicolumn{2}{|c|}{$\begin{array}{ll}\text { LAT } & \text { nbSPO }\end{array}$} \\
\hline Definition & $\begin{array}{l}\text { Area under } \\
\text { sporulating } \\
\text { progress curve }\end{array}$ & $\begin{array}{l}\text { Latent period, i.e. estimation of } \\
\text { the time elapsed from } \\
\text { inoculation to appearance of the } \\
\text { first pycnidia }\end{array}$ & $\begin{array}{l}\text { Final number of } \\
\text { pycnidiospores }^{b} \text { produced } \\
\text { per unit sporulating } \text { area }^{\mathrm{a}}\end{array}$ \\
\hline Measurement & Sporulating area ${ }^{a}$ & 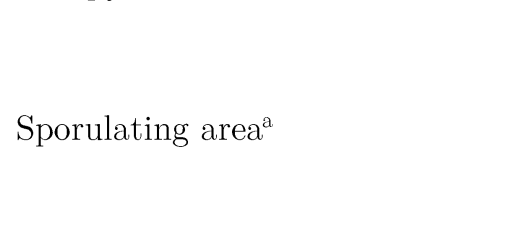 & $\begin{array}{l}\text { Total number of } \\
\text { pycnidiospores }^{\mathrm{b}} \text { collected in } \\
\text { lesion washing suspensions } \\
\text { and counted in a Malassez } \\
\text { counting chamber }\end{array}$ \\
\hline Calculation & $\begin{array}{l}\text { Midpoint rule } \\
\text { (trapezoidal integration } \\
\text { method) }\end{array}$ & $\begin{array}{l}\text { Number of days post-inoculation } \\
\text { required to reach } 5 \% \text { of the } \\
\text { maximum sporulating area }\end{array}$ & $\begin{array}{l}\text { Total number of } \\
\text { pycnidiospores }^{b} \text { divided by } \\
\text { maximum sporulating area }\end{array}$ \\
\hline \multirow{2}{*}{\multicolumn{4}{|c|}{$\begin{array}{l}\text { a leaf area covered by pycnidia (asexual fruiting bodies) } \\
\text { b asexual spores liberated from pycnidia } \\
{ }^{c} \text { suspensions obtained by sweeping the leaf sporulating area with a paintbrush into a fixed volume of water } \\
\text { Table } 2 \text { Impact of experimental variables on OD measurements across the experiment. We } \\
\text { investigated the experimental factors which can affect OD values, by fitting a generalized linear model to } \\
\text { growth kinetics data over a four-day period, during which we monitored spore concentration and optical } \\
\text { properties. The main effects and interactions with time and temperature are summarised by } P \text {-values }(\mathrm{F} \text { - } \\
\text { test). }\end{array}$}} \\
\hline & & & \\
\hline \multicolumn{2}{|l|}{ Variables } & $\begin{array}{l}\text { Effect on } \\
\text { OD }\end{array} \quad$ Interaction with time & nteraction with temperature \\
\hline \multicolumn{2}{|c|}{ Number of spores } & $<0.01$ & - \\
\hline \multicolumn{2}{|c|}{ Time } & $<0.01$ & - \\
\hline \multicolumn{2}{|l|}{ Temperature } & $<0.01$ & - \\
\hline \multicolumn{2}{|l|}{ Spore length } & 0.93 & 0.17 \\
\hline \multicolumn{2}{|l|}{ Spore width } & 0.12 & 0.19 \\
\hline \multicolumn{2}{|l|}{ Spore shape } & 0.73 & 0.37 \\
\hline \multicolumn{2}{|c|}{ Spore melanisation } & $<0.01$ & 0.07 \\
\hline \multicolumn{2}{|c|}{$\begin{array}{l}\text { Presence of hyphal growth } \\
\text { forms }\end{array}$} & 0.42 & $0.02 *$ \\
\hline
\end{tabular}

* The corresponding post-hoc test indicates that the limited presence of hyphal growth forms affected OD values only at $9.5^{\circ} \mathrm{C}$ (at which temperature there were more pseudohyphae $i$.e. growth forms presenting the first signs of branching of the filamentous structures). This interaction was not significant at the other 11 temperatures. 
Table 3 Characteristics and proportions of the four thermotypes identified in HCPC analysis (French $Z$. tritici populations; $\mathbf{n}=\mathbf{6 6}$ strains). Thermal responses were clustered on the basis of the following four TPC parameters: $\mathrm{P}_{\max }, \mathrm{T}_{\mathrm{opt}}, \mathrm{T}_{\max }$ and $\mathrm{TPB}_{80}$. $\mathrm{T}_{\max }$ does not appear in the table as its value was largely conserved across $Z$. tritici strains (no variation between clusters). Numbers in brackets indicate the value for the barycentre of each thermotype (corresponding to an HCPC cluster) (see Fig. 6b). Intercluster variations are summarised as follows, based on a statistical comparison with the overall mean of each parameter: '-' no deviation; ' $\boldsymbol{~ ' ~ h i g h e r ~ v a l u e ; ~ ' ~}$ identifies the TPC parameter characterising each thermotype most appropriately. The frequency of each thermotype is provided across (overall proportion) and within (occurrence within populations) the six sampled populations.

\begin{tabular}{|c|c|c|c|c|c|}
\hline \multirow{2}{*}{ Thermotype } & \multicolumn{3}{|c|}{ TPC parameters } & \multirow{2}{*}{$\begin{array}{c}\text { Overall } \\
\text { proportion } \\
(\%)\end{array}$} & \multirow{2}{*}{$\begin{array}{l}\text { Occurrence within } \\
\text { populations (ratio) }\end{array}$} \\
\hline & $\mathrm{P}_{\max }$ & $\mathrm{T}_{\text {opt }}$ & $\mathrm{TPB}_{80}$ & & \\
\hline Th1 & $0.24(\searrow)$ & $22.0(\nearrow)$ & $13.7(-)$ & 18.2 & $5 / 6$ \\
\hline Th2 & $0.28(-)$ & $20.4(-)$ & $13.1(\searrow)$ & 31.8 & $5 / 6$ \\
\hline Th3 & $0.32(\nearrow)$ & $18.5(\searrow \searrow)$ & $13.7(-)$ & 24.2 & $6 / 6$ \\
\hline Th4 & $0.28(-)$ & $19.8(\searrow)$ & $15.5(\nearrow)$ & 25.8 & $6 / 6$ \\
\hline
\end{tabular}




\section{NOTES}

\section{ACKNOWLEDGEMENTS}

We would like to thank Alain Fortineau for designing the experimental setup used for monitoring liquid medium and wheat leaf temperatures; Marc-Henri Lebrun for kindly providing the GFP-transformed strain of Z tritici; Laurent Falchetto, Bernard Gesret, Henriette Goyeau, Marc Leconte and Ivan Sache for their help in sampling the French $Z$. tritici populations used in this study; Ons El Kamel for her assistance in collecting epidemiological data on wheat plants; Bérengère Dalmais for providing support for fluorescence microscopy.

\section{FUNDING}

This work was supported by a grant from the French National Research Agency (ANR) as part of the 'Investissements d'Avenir' programme (SEPTOVAR project; LabEx BASC; ANR-11LABX-0034) and by a PhD fellowship from the French Ministry of Education and Research (MESR) awarded to A.-L. B.

\section{ELECTRONIC SUPPLEMENTARY MATERIAL}

Supplementary information is available in the online version of the paper.

- ESM 1: Optimal culture conditions for monitoring Z. tritici growth at different temperatures

- ESM 2: List and characteristics of the 15 pre-selected mathematical models used to establish TPCs

- ESM 3: Monitoring of spore characteristics over a four-day thermal phenotyping experiment

- ESM 4: Temperature monitoring system in liquid culture medium in 96-well microtiter plates

- ESM 5: Sampling and composition of the six French Z. tritici populations

- ESM 6: Comparisons between turbidity and automated measurements of spore concentration

\section{AUTHOR CONTRIBUTIONS}

A.-L. B., M.C. and F.S. conceived and designed the study. Experiments were performed by A.-L. B., with the help of G.D. for method development and J.L. for in vitro growth monitoring of the French Z. tritici populations. A.-L. B. performed data analyses. A.-L. B., M.C. and F.S. wrote the manuscript. 


\section{REFERENCES}

1. Lindow SE, Brandl MT (2003) Microbiology of the phyllosphere. Applied and Environmental Microbiology 69:1875-1883. doi: 10.1128/AEM.69.4.1875-1883.2003

2. Chelle $M$ (2005) Phylloclimate or the climate perceived by individual plant organs: What is it? How to model it? What for? New Phytologist 166:781-790. doi: 10.1111/j.14698137.2005.01350.x

3. Angilletta MJ (2009) Thermal adaptation: a theoretical and empirical synthesis. Oxford University Press, Oxford

4. Bernard F, Sache I, Suffert F, Chelle M (2013) The development of a foliar fungal pathogen does react to leaf temperature! New Phytologist 198:232-240. doi: 10.1111/nph.12134

5. Sutherst RW, Constable F, Finlay KJ, Harrington R, Luck J, Zalucki MP (2011) Adapting to crop pest and pathogen risks under a changing climate. Wiley Interdisciplinary Reviews: Climate Change 2:220-237. doi: 10.1002/wcc.102

6. Boyd PW, Rynearson TA, Armstrong EA, et al (2013) Marine phytoplankton temperature versus growth responses from polar to tropical waters - outcome of a scientific community-wide study. PLoS ONE 8:e63091. doi: 10.1371/journal.pone.0063091

7. Huey RB, Stevenson RD (1979) Integrating thermal physiology and ecology of ectotherms: a discussion of approaches. American Zoologist 19:357-366. doi: 10.1093/icb/19.1.357

8. Sinclair BJ, Marshall KE, Sewell MA, et al (2016) Can we predict ectotherm responses to climate change using thermal performance curves and body temperatures? Ecology Letters 19:1372-1385. doi: 10.1111/ele.12686

9. Low-Décarie E, Boatman TG, Bennett N, Passfield W, Gavalás-Olea A, Siegel P, Geider RJ (2017) Predictions of response to temperature are contingent on model choice and data quality. Ecology and Evolution 7:10467-10481. doi: 10.1002/ece3.3576

10. Zhan J, McDonald BA (2011) Thermal adaptation in the fungal pathogen Mycosphaerella graminicola. Molecular Ecology 20:1689-1701. doi: 10.1111/j.1365294X.2011.05023.x

11. Wiser MJ, Lenski RE (2015) A comparison of methods to measure fitness in Escherichia coli. PLoS ONE 10:e0126210. doi: 10.1371/journal.pone.0126210

12. Angilletta MJ (2006) Estimating and comparing thermal performance curves. Journal of Thermal Biology 31:541-545. doi: 10.1016/j.jtherbio.2006.06.002

13. Quinn BK (2017) A critical review of the use and performance of different function types for modeling temperature-dependent development of arthropod larvae. Journal of Thermal Biology 63:65-77. doi: 10.1016/j.jtherbio.2016.11.013

14. Eyal Z (1999) The Septoria tritici and Stagonospora nodorum blotch diseases of wheat. European Journal of Plant Pathology 105:629-641

15. Suffert F, Ravigné V, Sache I (2015) Seasonal changes drive short-term selection for fitness traits in the wheat pathogen Zymoseptoria tritici. Applied and Environmental Microbiology 81:6367-6379. doi: 10.1128/AEM.00529-15

16. Lendenmann MH, Croll D, Palma-Guerrero J, Stewart EL, McDonald BA (2016) QTL mapping of temperature sensitivity reveals candidate genes for thermal adaptation and growth morphology in the plant pathogenic fungus Zymoseptoria tritici. Heredity 116:384-394. doi: 10.1038/hdy.2015.111 
17. Pariaud B, Ravigné V, Halkett F, Goyeau H, Carlier J, Lannou C (2009) Aggressiveness and its role in the adaptation of plant pathogens. Plant Pathology 58(3):409-424. 18. Ahmed HU, Mundt CC, Hoffer ME, Coakley SM (1996) Selective influence of wheat cultivars on pathogenicity of Mycosphaerella graminicola (anamorph Septoria tritici). Phytopathology 86:454-458. doi: 10.1094/Phyto-86-454

19. Donlan RM (2002) Biofilms: microbial life on surfaces. Emerging Infectious Diseases 8:881890. doi: 10.3201/eid0809.020063

20. Hall BG, Acar H, Nandipati A, Barlow M (2014) Growth rates made easy. Molecular Biology and Evolution 31:232-238. doi: 10.1093/molbev/mst187

21. Burnham KP, Anderson DR (2004) Model selection and multimodel inference. Springer New York, New York

22. Jassby AD, Platt $T$ (1976) Mathematical formulation of the relationship between photosynthesis and light for phytoplankton: photosynthesis-light equation. Limnology and Oceanography 21:540-547. doi: 10.4319/lo.1976.21.4.0540

23. International Organization for Standardization (1994) Accuracy (trueness and precision) of measurement methods and results. ISO Standards catalogue

24. International Organization for Standardization (2017) Guidance for the use of repeatability, reproducibility and trueness estimates in measurement uncertainty evaluation. ISO Standards catalogue

25. Butler MJ, Day AW (1998) Fungal melanins: a review. Canadian Journal of Microbiology 44:1115-1136. doi: 10.1139/w98-119

26. Steinberg G (2015) Cell biology of Zymoseptoria tritici: pathogen cell organization and wheat infection. Fungal Genetics and Biology 79:17-23. doi: 10.1016/j.fgb.2015.04.002

27. Pigliucci M (2003) Phenotypic integration: studying the ecology and evolution of complex phenotypes. Ecology Letters 6:265-272. doi: 10.1046/j.1461-0248.2003.00428.x

28. Suffert F, Sache I, Lannou C (2013) Assessment of quantitative traits of aggressiveness in Mycosphaerella graminicola on adult wheat plants. Plant Pathology 62:1330-1341. doi: 10.1111/ppa.12050

29. Rousseeuw PJ (1987) Silhouettes: a graphical aid to the interpretation and validation of cluster analysis. Journal of Computational and Applied Mathematics 20:53-65. doi: 10.1016/0377-0427(87)90125-7

30. Abràmoff MD, Magalhães PJ, Ram SJ (2004) Image processing with ImageJ. Biophotonics International 11:36-42

31. R Core Team (2018) R: A language and environment for statistical computing. R Foundation for Statistical Computing, Vienna, Austria

32. Pinheiro J, Bates D, DebRoy S, Sarkar D (2018) nlme: linear and nonlinear mixed effects models. Comprehensive R Archive Network

33. Lê S, Josse J, Husson F (2008) FactoMineR: an R package for multivariate analysis. Journal of Statistical Software 25:1-18. doi: 10.18637/jss.v025.i01

34. Hennig C (2010) fpc: flexible procedures for clustering. Comprehensive R Archive Network 35. Gauthier GM (2015) Dimorphism in fungal pathogens of mammals, plants, and insects. PLoS Pathogens 11:e1004608. doi: 10.1371/journal.ppat.1004608 
36. Linde CC, Zhan J, McDonald BA (2002) Population structure of Mycosphaerella graminicola: from lesions to continents. Phytopathology 92:946-955. doi: 10.1094/PHYTO.2002.92.9.946

37. Zhan J, Linde CC, Jurgens T, Merz U, Steinebrunner F, McDonald BA (2005) Variation for neutral markers is correlated with variation for quantitative traits in the plant pathogenic fungus Mycosphaerella graminicola. Molecular Ecology 14:2683-2693. doi: 10.1111/j.1365-294x.2005.02638.x

38. Farine DR, Montiglio P-O, Spiegel O (2015) From individuals to groups and back: the evolutionary implications of group phenotypic composition. Trends in Ecology \& Evolution 30:609-621. doi: 10.1016/j.tree.2015.07.005

39. Van de Pol M (2012) Quantifying individual variation in reaction norms: how study design affects the accuracy, precision and power of random regression models. Methods in Ecology and Evolution 3:268-280. doi: 10.1111/j.2041-210x.2011.00160.x

40. Ferrandino FJ (2012) Time scales of inoculum production and the dynamics of the epidemic. Phytopathology 102:728-732. doi: 10.1094/PHYTO-03-12-0051-LE

41. Ghalambor CK, McKay JK, Carroll SP, Reznick DN (2007) Adaptive versus nonadaptive phenotypic plasticity and the potential for contemporary adaptation in new environments. Functional Ecology 21:394-407. doi: 10.1111/j.1365-2435.2007.01283.x

42. Zhan F, Xie Y, Zhu W, Sun D, McDonald BA, Zhan J (2016) Linear correlation analysis of Zymoseptoria tritici aggressiveness with in vitro growth rate. Phytopathology 106:1255-1261. doi: 10.1094/PHYTO-12-15-0338-R

43. Paisley D, Robson GD, Denning DW (2005) Correlation between in vitro growth rate and in vivo virulence in Aspergillus fumigatus. Medical Mycology 43:397-401. doi: $10.1080 / 13693780400005866$

44. Niehaus AC, Angilletta MJ, Sears MW, Franklin CE, Wilson RS (2012) Predicting the physiological performance of ectotherms in fluctuating thermal environments. Journal of Experimental Biology 215:694-701. doi: 10.1242/jeb.058032

45. Giraud T, Koskella B, Laine A-L (2017) Introduction: microbial local adaptation: insights from natural populations, genomics and experimental evolution. Molecular Ecology 26:1703-1710. doi: 10.1111/mec.14091

46. Bazakos C, Hanemian M, Trontin C, Jiménez-Gómez JM, Loudet O (2017) New strategies and tools in quantitative genetics: how to go from the phenotype to the genotype. Annual Review of Plant Biology 68:435-455. doi: 10.1146/annurev-arplant-042916-040820

47. Brown SDM, Wurst W, Kühn R, Hancock JM (2009) The functional annotation of mammalian genomes: the challenge of phenotyping. Annual Review of Genetics 43:305-333. doi: 10.1146/annurev-genet-102108-134143

48. Reed TE, Schindler DE, Waples RS (2011) Interacting effects of phenotypic plasticity and evolution on population persistence in a changing climate. Conservation Biology 25:56-63. doi: $10.1111 / \mathrm{j} .1523-1739.2010 .01552 . \mathrm{x}$

49. Zhan J, McDonald BA (2013) Field-based experimental evolution of three cereal pathogens using a mark-release-recapture strategy. Plant Pathology 62:106-114. doi: $10.1111 /$ ppa. 12130

50. Fisher KJ, Lang GI (2016) Experimental evolution in fungi: an untapped resource. Fungal Genetics and Biology 94:88-94. doi: 10.1016/j.fgb.2016.06.007 
51. Chevin L-M, Lande R, Mace GM (2010) Adaptation, plasticity, and extinction in a changing environment: towards a predictive theory. PLoS Biology 8:e1000357. doi: 10.1371/journal.pbio. 1000357 